including the effect of a brief occupational health history training program.

Methods All chiropractic interns at one clinic location completed questionnaires assessing their attitudes and perceptions regarding documenting occupational history of their patients. Each intern enrolled in the study for two or more trimesters also participated in an hour-long training session on taking an occupational history. The supervising clinician independently evaluated charting behaviours of interns for the duration of the study.

Result Twenty interns participated for 4 to 12 months. The supervising clinician assessed the interns' level of documenting occupational history for 202 new patient or re-examination visits. Patient's current occupation was documented in $93 \%$ of these visits, but a detailed occupational history was documented in only $11 \%$ of these visits despite the chief complaint being related to their occupation 39\% of the time. After the first group of interns completed training, documentation of the relationship between occupation and chief complaint increased from $20 \%$ of visits to $57 \%$. When interns assessed their own recordkeeping practices, all interns reported asking about current occupation for most patients, but indicated their other occupational history taking and documentation behaviours can vary.

Discussion While documenting current occupation was relatively high among the chiropractic interns, additional detailed occupational information was not usually included in their documentation. Additional training on occupational history taking did not substantially change those behaviours, but did increase the interns relating the chief complaint to the patient's work.

\section{CHRONIC MUSCULOSKELETAL PAIN: CONNECTING THE DOTS}

1,2Suparna Damany. 'Damany Centre For Chronic Pain And Holistic Well-being, Allentown, USA; ${ }^{2}$ Adjunct professor, DeSales University, Bethlehem, Pennsylvania, USA

\subsection{6/oemed-2018-ICOHabstracts.297}

4Chronic musculoskeletal pain is the disorder of our decade, and frustrating for both the patient as well as the healthcare practitioner. A cause for this frustration is an inability to understand the inter-relationships between the different bodily systems involved. An understanding of the pathophysiology of chronic musculoskeletal pain will allow for development of a comprehensive and effective treatment plan.

In this presentation, a case study will elucidate the 'pieces of the puzzle' in a chronic musculoskeletal injury. The symptoms will be related to the pathophysiology and the effectiveness of a logical conservative and comprehensive treatment plan will be demonstrated.

Application of this protocol to the general chronic pain population will be discussed.
1530

PROFESSIONALS COMPETENCES FOR WORKER HEALTH SURVEILLANCE

${ }^{1}$ VA Mininel*, ${ }^{2} \mathrm{~L}$ Geraldi, ${ }^{3} \mathrm{FM}$ Miranda, ${ }^{4}$ AP Griggio, ${ }^{1} \mathrm{JAM}$ Silva, ${ }^{5} \mathrm{~S}$ Appenzeller. ${ }^{1}$ Assistance Professor, Federal University of Sao Carlos, Sao Carlos, Brazil;, ${ }^{2}$ Undergraduate student, Federal University of Sao Carlos, Sao Carlos, Brazil; ${ }^{3}$ Young researcher, Federal University of Sao Carlos, Sao Carlos, Brazil; ' ${ }^{4}$ Master's student, Federal University of Sao Carlos, Sao Carlos, Brazil; ${ }^{5}$ Associate Professor, State University of Campinas, Campinas, Brazil

\subsection{6/oemed-2018-ICOHabstracts.298}

Introduction General and specifics professional competencies aim to guide the professional profile expected and must to address health services needs and demands. Professional competences to worker health surveillance in a perspective of comprehensive health care in the health network (and not as a medicine specialty) are not well known in the literature, what compromise education process and health care assistance. Thus, this study aimed to identify professional competences to worker health surveillance.

Methods Qualitative research with triangulation of data, performed in three steps:

1. documental analysis of Brazilian National Curriculum Standards for the healthcare field and pedagogical projects of seven courses from Federal University of Sao Carlos: nursing, medicine, physiotherapy, occupational therapy, psychology, gerontology and physical education.

2. Systematic review was conducted in databases: Latin American and Caribbean Health Sciences Literature, MEDLINE/PubMed, Web of Science, Scopus and Education Resources Information Centre. Gathering strategies includes MESH terms: occupational health, curriculum, competencybased education and undergraduate medical education.

3. Interview with professors from seven courses of university and professionals of healthcare system, guided by criteria of saturation data and thematic analysis of data.

Results Preliminary results show general competencies from undergraduate courses of health area that are related to worker health surveillance, as communication, team work, leadership, health management skills and health education. Documental analysis demonstrated several specific competences from each profession, but none related to worker health surveillance - what should include health promotion, risks, diseases and harm prevention, professional rehabilitation and return to work. Some pedagogical projects presented content that exploit different perspectives of workers health and safety, but not in an integrated way or represented by competences. Discussion Preliminary results show that education standards, pedagogical projects and literature review still consider worker health as occupational health, i.e., as a specialty, not exploring this theme as whole in undergraduate education. 


\section{INTERPROFESSIONAL EDUCATION FOR WORKER} HEALTH SURVEILLANCE

${ }^{1}$ VA Mininel ${ }^{*},{ }^{2}$ AP Griggio, ${ }^{1}$ JAM Silva, ${ }^{3} S$ Appenzeller. ${ }^{1}$ Assistance Professor, Federal University of Sao Carlos, Sao Carlos, Brazil; ${ }^{2}$ Master's student, Federal University of Sao Carlos, Sao Carlos, Brazil; ${ }^{3}$ Associate Professor, State University of Campinas, Campinas, Brazil

\subsection{6/oemed-2018-ICOHabstracts.299}

Introduction Interprofessional education and collaborative practice are an innovative strategy to strengthen healthcare services and have been encouraged by World Health Organisation. Efforts to promote them in undergraduate and professional education can contribute to promoting comprehensive health care and to answering healthcare needs and demands. Considering the relevance of this thematic and its potential contribution for worker health surveillance, this study aimed to develop a Interprofessional education activity to undergraduate students and healthcare professionals to promote actions of worker health surveillance.

Methods Intervention study, performed in two steps in a Brazilian university. (1) planning of the activity, through the collaborative practice among professors from seven courses from health area: nursing, medicine, physiotherapy, occupational therapy, gerontology, phycology and physical education. Meetings were guided by Interprofessional education and aimed to construct common competencies for multiple professions, to define pedagogical strategies, learning goals and evaluation tools. (2) implementation of the 60 hours-activity, which will be offer to undergraduate students from different health courses and healthcare professional in the next semester. Study approved by ethical committee of research.

Results Collaborative practice and interprofessional education are themes less known and explored in Brazilian universities and healthcare services context. Efforts in this way had been made in our setting to allow the planning of activity. It has been an important step in the university bring these theoretical approach to discussion, reflection and learning and, thus, changing the reality through an activity that has potential to become part of regular schedule of optative activities.

Discussion Until this moment of research, the activity has been planned and the collaborative practice has been possible, promoting actions of interprofessional educational and strengthen the healthcare services.

\section{PREPARED FOR WORKING LIFE!}

P Naumanen* ${ }^{*}$ J Liesivuori. University of Turku, Turku, Finland

\subsection{6/oemed-2018-ICOHabstracts.300}

Introduction There is a lack of systematic education about wellbeing at work to prepare students for working life in Finland. The aim of this project is to prepare students for working life so that they have positive attitude toward working life and have certain personal and professional abilities as well as abilities to develop their personal and work community wellbeing at work. The future goal is that new generation will have better abilities to develop wellbeing at work, which is the way to improve quality of working life and to create a new wellbeing culture to workplaces.

The project will be implemented during 1 st November, 2015 and 31 st October, 2018 managed by University of Turku, Finland, and co-operated with Universities of Oulu,
Jyväskylä, Vaasa and Lappland with University of Applied Sciences of Oulu, Jyväskylä, Vaasa, Saimaa, and with the Oulu Vocational College. This project will support national and regional co-operation between universities and other institutes.

Methods Project activities will be planned and implemented together with educational personnel for different groups of students, who will start their working life. The education will reach thousands of students every year.

Common activities for all project organisations are to build network based wellbeing at work education for students, to organise general and student seminars, to build professional and organisational networks, and to conduct student thesis. Wellbeing at work lectures will be also offered to those educational institutes that are not participating in this project.

Result Expected long term future results are more positive climate and better quality of working life, which hopefully will affect to increase productivity and decrease health costs as wel

\section{MANAGING HEALTH AND SAFETY IN THE HEALTHCARE SETTING - AN E-LEARNING TRAINING PROGRAMME}

Nicholas J Parkinson*. Health Service Executive, National Health and Safety Function

\subsection{6/oemed-2018-ICOHabstracts.301}

Introduction The National Health and Safety Function have developed an online training programme entitled 'Managing Health and Safety in the Healthcare Setting'. The programme provides managers with the appropriate information and guidance on the core responsibilities and actions required to implement a safety management programme within their area of remit.

This programme is mandatory for HSE managers, department heads and all those with responsibility for safety health and wellbeing (30 000 staff approx).

Under the Safety, Health and Welfare at Work Act 2005 it is the responsibility of a manager to manage safety, health and welfare in the workplace and ensure, so far as is reasonably practicable, the safety, health and welfare of employees and others in the workplace.

Methods The aim of the programme is to provide managers with the information and support to enable them to manage safety, health and welfare and implement a safety management programme within their area of responsibility as far as is reasonably practicable.

Result We developed an innovative E-Learning training programme available to all levels of HSE Management on 'Managing Health and Safety in the Healthcare Setting' (available on HSELaND).

On completion of this programme staff will be deemed competent in:

- Describing their role within the legal and policy context with regard to health and safety in the healthcare

- Identifying best practice with regard to the implementation of a Safety Management Programme

- Understanding the purpose and importance of Site Specific Safety Statement (SSSS)

- Implementing a structured approach to Risk Assessment.

Discussion Managers have significant legal duties with regard to management of Occupational Safety and Health. This programme provides a key component in our blended learning strategy. 